PROCEEDINGS OF THE

AMERICAN MATHEMATICAL SOCIETY

Volume 133, Number 2, Pages 349-355

S 0002-9939(04)07453-2

Article electronically published on September 2, 2004

\title{
A NOTE ON PRINCIPAL PARTS ON PROJECTIVE SPACE AND LINEAR REPRESENTATIONS
}

\author{
HELGE MAAKESTAD
}

(Communicated by Michael Stillman)

\begin{abstract}
Let $H$ be a closed subgroup of a linear algebraic group $G$ defined over a field of characteristic zero. There is an equivalence of categories between the category of linear finite-dimensional representations of $H$, and the category of finite rank $G$-homogeneous vector bundles on $G / H$. In this paper we will study this correspondence for the sheaves of principal parts on projective space, and we describe the representation corresponding to the principal parts of a line bundle on projective space.
\end{abstract}

\section{INTRODUCTION}

In this note we will study the vector bundles of principal parts $\mathcal{P}^{k}(\mathcal{O}(n))$ of a line bundle on projective space over a field $F$ of characteristic zero from a representation theoretic point of view. We consider projective $N$-space as a quotient $S L(V) / P$, where $V$ is an $(N+1)$-dimensional vector space over $F$, and $P$ is the subgroup of $S L(V)$ stabilizing a line $L$ in $V$. There is an equivalence of categories between the category of finite rank $S L(V)$-homogeneous vector bundles on $S L(V) / P=\mathbf{P}\left(V^{*}\right)$ and the category of linear finite-dimensional representations of $P$. The principal parts $\mathcal{P}^{k}(\mathcal{O}(n))$ are $S L(V)$-homogeneous vector bundles on $\mathbf{P}\left(V^{*}\right)$, and the novelty of this note is that we describe the $P$-representation corresponding to the principal parts. The main result is Theorem 2.4, which says the following: Let $L^{*}$ be the dual of the $P$-module $L$. Then for all $1 \leq k<n$, the $P$-representation corresponding to $\mathcal{P}^{k}(\mathcal{O}(n))$ is $S^{n-k}\left(L^{*}\right) \otimes S^{k}\left(V^{*}\right)$. As a corollary, we obtain the splitting type of $\mathcal{P}^{k}(\mathcal{O}(n))$ on $\mathbf{P}\left(V^{*}\right)$ for all $1 \leq k<n$, and recover results obtained in [6], 7], [8] and $[9]$.

\section{PRINCIPAL PARTS ON PROJECTIVE SPACE}

In this section we give the representation corresponding to $\mathcal{P}^{k}(\mathcal{O}(n))$ on $\mathbf{P}\left(V^{*}\right)$ for all $1 \leq k<n$, where $V$ is an $F$-vector space of dimension $N+1$ and $F$ is a field of characteristic 0 . A variety is an integral scheme of finite type over $F$.

Received by the editors May 16, 2002 and, in revised form, July 7, 2003.

2000 Mathematics Subject Classification. Primary 14L30, 20 C15.

Key words and phrases. Homogeneous spaces, homogeneous vector bundles, principal parts, linear representations, splitting type.

This work was partially supported by the Emmy Noether Research Institute for Mathematics, the Minerva Foundation of Germany, the Excellency Center "Group Theoretic Methods in the Study of Algebraic Varieties" of the Israel Science Foundation and the EAGER Foundation (EU network, HPRN-CT-2000-00099). 
We will consider closed points when we talk about points of a scheme. Let $V$ be a finite-dimensional vector space over $F$. We let $G L(V)$ denote the group of all invertible linear transformations of $V$. It is an algebraic group in the sense of [2], Chapt. 1. A linear algebraic group is a closed subgroup of $G L(V)$. Let $S L(V)$ be the linear algebraic group of linear transformations of $V$ with determinant 1 . Let $L$ in $V$ be a line, and $P$ the closed subgroup of $S L(V)$ stabilizing $L$. Then the quotient $S L(V) / P$ (which exists by 2, Theorem 6.8) is isomorphic to $\mathbf{P}\left(V^{*}\right)$, the projective space of lines in $V$ (see [1], Section 4.2). This works over any field, not only the complex numbers. There exists a natural left $S L(V)$-action on $\mathbf{P}\left(V^{*}\right)$, making it into a homogeneous space for $S L(V)$. Also by [1], Chapt. 4, there exists an equivalence of categories between the category of finite rank homogeneous vector bundles on $S L(V) / P$ and the category of linear finite-dimensional representations of $P$, and under this correspondence the dimension of the representation gives the rank of the corresponding vector bundle. Hence any character of $P$ gives a homogeneous line bundle on $S L(V) / P$. The line $L$ corresponds to a character of $P$, and the bundle corresponding to the dual line $L^{*}$ is the line bundle $\mathcal{O}(1)$ on $\mathbf{P}\left(V^{*}\right)$ (see [1, Section 4.2). It is also a standard fact that any linear finite-dimensional representation $\rho$ of $P$ lifting to a representation $\tilde{\rho}$ of $S L(V)$ corresponds to a trivial abstract vector bundle on $\mathbf{P}\left(V^{*}\right)$. There exists on any scheme an equivalence of categories between the category of locally free finite rank sheaves and the category of finite rank vector bundles; hence we will use these two notions interchangeably.

Pick a basis $e_{0}, \ldots, e_{N}$ for $V$. Let $x_{0}, \ldots, x_{N}$ be the dual basis, and let $L$ be the line spanned by $e_{0}$. Having chosen a basis for $V$, it follows that $S L(V)$ may be identified with the group of square rank $N+1$ matrices with determinant equal to 1. The group $P$ may be identified with the subgroup of $S L(V)$ consisting of matrices $g$ of the form

$$
g=\left(\begin{array}{cccc}
a & * & \cdots & * \\
0 & a_{11} & \cdots & a_{1 n} \\
\vdots & \vdots & \vdots & \vdots \\
0 & a_{n 1} & \cdots & a_{n n}
\end{array}\right) .
$$

The one-dimensional representation $\chi: P \rightarrow G L\left(S^{n}\left(L^{*}\right)\right)$ corresponding to the line bundle $\mathcal{O}(n)$ is given by $\chi(g)=a^{-n}$.

Let $X$ be a smooth variety of dimension $d$ and consider the diagonal $\Delta$ in $X \times X$. Let $\mathcal{I}$ be the sheaf of ideals of $\mathcal{O}_{X \times X}$ defining the diagonal $\Delta$, and define $\mathcal{O}_{\Delta^{k}}$ to be $\mathcal{O}_{X \times X} / \mathcal{I}^{k+1}$.

Definition 2.1. Let $p, q$ be the projection maps from $X \times X$ to $X$, and let $\mathcal{E}$ be an $\mathcal{O}_{X}$-module. Define $\mathcal{P}^{k}(\mathcal{E})=p_{*}\left(\mathcal{O}_{\Delta^{k}} \otimes q^{*} \mathcal{E}\right)$ to be the $k$ th order principal parts of the module $\mathcal{E}$. We put $\mathcal{P}^{k}\left(\mathcal{O}_{X}\right)=\mathcal{P}^{k}$.

Note that by [6], if the rank of $\mathcal{E}$ is $e, \mathcal{P}^{k}(\mathcal{E})$ is a vector bundle of rank $e\left(\begin{array}{c}d+k \\ d\end{array}\right)$ on $X$. Assume that $G$ is an algebraic group, and that $X$ is a homogeneous space for $G$. Assume furthermore that $\mathcal{E}$ is a $G$-homogeneous vector bundle on $X$; then it follows that $\mathcal{P}^{k}(\mathcal{E})$ is again a $G$-homogeneous vector bundle on $X$. Consider the line bundle $\mathcal{O}(n)$ on $\mathbf{P}\left(V^{*}\right)=S L(V) / P$; then $\mathcal{O}(n)$ is an $S L(V)$-homogeneous line bundle on $\mathbf{P}\left(V^{*}\right)$ for all $n$, and we may consider the $S L(V)$-homogeneous vector bundle $\mathcal{P}^{k}(\mathcal{O}(n))$. We want to compute the representation $\rho$ of $P$ corresponding to the homogeneous vector bundle $\mathcal{P}^{k}(\mathcal{O}(n))$ for all $1 \leq k<n$ on $\mathbf{P}\left(V^{*}\right)$. Let, in the following, $X=\mathbf{P}\left(V^{*}\right)$ and consider the projection maps $p, q$ from $X \times X$ to 
$X$. Let $\mathcal{I}$ in $\mathcal{O}_{X \times X}$ be the ideal of the diagonal. We have an exact sequence of $S L(V)$-homogeneous vector bundles on $X \times X$ :

$$
0 \rightarrow \mathcal{I}^{k+1} \rightarrow \mathcal{O}_{X \times X} \rightarrow \mathcal{O}_{\Delta^{k}} \rightarrow 0 .
$$

Apply the functor $p_{*}\left(-\otimes q^{*} \mathcal{O}(n)\right)$ to the sequence (2.1.1) to get a long exact sequence

$$
\begin{gathered}
0 \rightarrow p_{*}\left(\mathcal{I}^{k+1} \otimes q^{*} \mathcal{O}(n)\right) \rightarrow p_{*} q^{*} \mathcal{O}(n) \rightarrow \mathcal{P}^{k}(\mathcal{O}(n)) \\
\rightarrow R^{1} p_{*}\left(\mathcal{I}^{k+1} \otimes q^{*} \mathcal{O}(n)\right) \rightarrow R^{1} p_{*} q^{*} \mathcal{O}(n) \rightarrow R^{1} p_{*}\left(\mathcal{O}_{\Delta^{k}} \otimes q^{*} \mathcal{O}(n)\right) \rightarrow \cdots
\end{gathered}
$$

of vector bundles. The sequence (2.1.2) is a sequence of vector bundles because all sheaves in the sequence are coherent, and it is a standard fact that a homogeneous coherent sheaf on a homogeneous space is locally free. Since the sequence (2.1.2) is a sequence of vector bundles, we get an exact sequence of $P$-representations when we pass to the fiber at $\bar{e}$.

Consider the diagram

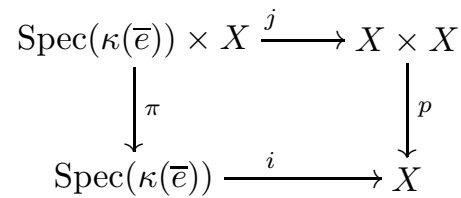

Then by [5], Chapt. III, Sect. 12, we get maps

$$
\phi^{i}: R^{i} p_{*}\left(\mathcal{I}^{k+1} \otimes q^{*} \mathcal{O}(n)\right)(\bar{e}) \rightarrow R^{i} \pi_{*}\left(j^{*}\left(\mathcal{I}^{k+1} \otimes q^{*} \mathcal{O}(n)\right)\right)
$$

of $\mathcal{O}_{X}$-modules. Put, for any $\mathcal{O}_{X \times X}$-module $\mathcal{E}$,

$$
h^{i}(y, E)=\operatorname{dim}_{\kappa(y)} \mathrm{H}^{i}\left(X_{y}, \mathcal{E}_{y}\right),
$$

where $X_{y}$ is the fiber $p^{-1}(y)$ and $\mathcal{E}_{y}$ is the restriction of $\mathcal{E}$ to $X_{y}$. We see that

$$
h^{i}\left(y, \mathcal{I}^{k+1} \otimes q^{*} \mathcal{O}(n)\right)=\operatorname{dim}_{\kappa(y)} \mathrm{H}^{i}\left(X, \mathfrak{m}_{y}^{k+1} \otimes \mathcal{O}(n)\right)
$$

is a constant function of $y$ for $i=0,1, \ldots$ for the following reason: Consider the commutative diagram

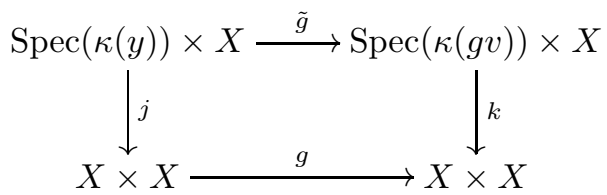

where the action of $S L(V)$ on $X \times X$ is given by $g(x, y)=(g x, g y)$. In general, if $G \times Y \rightarrow^{\sigma} Y$ is an algebraic group acting on a scheme $Y$, and $\mathcal{E}$ is a $G$-linearized sheaf on $Y$, then there exists an isomorphism $I: \sigma^{*} \mathcal{E} \rightarrow p^{*} \mathcal{E}$, where $p: G \times Y \rightarrow Y$ is the projection map. It follows that for all $g \in G$ we get an isomorphism $g^{*} \mathcal{E} \cong \mathcal{E}$ of sheaves. Then, since $\mathcal{O}(n)$ and $\mathcal{I}^{k+1} \otimes q^{*} \mathcal{O}(n)$ are $S L(V)$-homogeneous sheaves, we have an isomorphism

$$
\tilde{g}^{*}\left(\mathfrak{m}_{g y}^{k+1} \otimes \mathcal{O}(n)\right)=j^{*} g^{*}\left(\mathcal{I}^{k+1} \otimes q^{*} \mathcal{O}(n)\right)=\mathfrak{m}_{y}^{k+1} \otimes \mathcal{O}(n) ;
$$

hence since $\tilde{g}$ is an isomorphism, we see that we have an isomorphism

$$
\mathfrak{m}_{y}^{k+1} \otimes \mathcal{O}(n) \cong \mathfrak{m}_{g y}^{k+1} \otimes \mathcal{O}(n)
$$


of sheaves for all $g$ in $S L(V)$. It follows that

$$
\operatorname{dim}_{\kappa(y)} \mathrm{H}^{i}\left(X, \mathfrak{m}_{y}^{k+1} \otimes \mathcal{O}(n)\right)=\operatorname{dim}_{\kappa(g y)} \mathrm{H}^{i}\left(X, \mathfrak{m}_{g y}^{k+1}, \mathcal{O}(n)\right)
$$

for all $g$ in $S L(V)$; hence by [5], Chapt. III, Corr. 12.9, it follows that the maps $\phi^{i}$ are isomorphisms for $i=0,1, \ldots$ Here $\mathfrak{m}_{y}$ is the sheaf of ideals corresponding to the point $y$ in $X$. We get an exact sequence

$$
\begin{aligned}
0 & \rightarrow \mathrm{H}^{0}\left(X, \mathcal{O}(n) \otimes \mathfrak{m}_{\bar{e}}^{k+1}\right) \rightarrow \mathrm{H}^{0}(X, \mathcal{O}(n)) \rightarrow \mathcal{P}^{k}(\mathcal{O}(n))(\bar{e}) \\
& \rightarrow \mathrm{H}^{1}\left(X, \mathcal{O}(n) \otimes \mathfrak{m}_{\bar{e}}^{k+1}\right) \rightarrow \mathrm{H}^{1}(X, \mathcal{O}(n)) \rightarrow \cdots
\end{aligned}
$$

of $P$-representations.

Lemma 2.2. For all $1 \leq k<n$ we have that $\mathrm{H}^{1}\left(X, \mathcal{O}(n) \otimes \mathfrak{m}_{\bar{e}}^{k+1}\right)=0$.

Proof. Consider the exact sequence (2.1.3). We prove that

$$
\operatorname{dim}_{F} \mathrm{H}^{0}(X, \mathcal{O}(n))-\operatorname{dim}_{F} \mathrm{H}^{0}\left(X, \mathcal{O}(n) \otimes \mathfrak{m}_{\bar{e}}^{k+1}\right)=\operatorname{dim}_{F} \mathcal{P}^{k}(\mathcal{O}(n))(\bar{e}),
$$

and then the result follows by counting dimensions. We have that $\mathrm{H}^{0}(X, \mathcal{O}(n))$ equals $S^{n}\left(V^{*}\right)$, where $V^{*}$ is the $F$-vector space on the basis $x_{0}, \ldots, x_{N}$. We also see that $\mathrm{H}^{0}\left(X, \mathcal{O}(n) \otimes \mathfrak{m}_{\bar{e}}^{k+1}\right)$ equals $m^{k+1} S^{n-(k+1)}\left(V^{*}\right)$ considered as a subvectorspace of $S^{n}\left(V^{*}\right)$. Here $m$ is the $F$-vector space on the basis $x_{1}, \ldots, x_{N}$ and $m^{k+1} S^{n-(k+1)}\left(V^{*}\right)$ is the image of the natural map

$$
S^{k+1}(m) \otimes S^{n-(k+1)}\left(V^{*}\right) \rightarrow S^{n}\left(V^{*}\right) .
$$

Write $V^{*}$ as the direct sum $F x_{0} \oplus m$. Then it follows that

$$
m^{k+1} S^{n-(k+1)}\left(V^{*}\right)=x_{0}^{n-(k+1)} m^{k+1} \oplus \cdots \oplus x_{0} m^{n-1} \oplus m^{n} ;
$$

hence we see that the dimension of $m^{k+1} S^{n-(k+1)}\left(V^{*}\right)$ equals $\sum_{i=k+1}^{n}\left(\begin{array}{c}i+N-1 \\ N-1\end{array}\right)$. We also see that the dimension of $S^{n}\left(V^{*}\right)$ equals $\sum_{i=0}^{n}\left(\begin{array}{c}i+N-1 \\ N-1\end{array}\right)$, and it follows that

$$
\operatorname{dim}_{F} S^{n}\left(V^{*}\right)-\operatorname{dim}_{F} m^{k+1} S^{n-(k+1)}\left(V^{*}\right)=\sum_{i=0}^{k}\left(\begin{array}{c}
i+N-1 \\
N-1
\end{array}\right) .
$$

It follows that

$$
\sum_{i=0}^{k}\left(\begin{array}{c}
i+N-1 \\
N-1
\end{array}\right)=\left(\begin{array}{c}
k+N \\
N
\end{array}\right)=\operatorname{dim}_{F} \mathcal{P}^{k}(\mathcal{O}(n))(\bar{e}),
$$

and we have proved that

$$
\operatorname{dim}_{F} \mathrm{H}^{0}(X, \mathcal{O}(n))-\operatorname{dim}_{F} \mathrm{H}^{0}\left(X, \mathcal{O}(n) \otimes \mathfrak{m}_{\bar{e}}^{k+1}\right)=\operatorname{dim}_{F} \mathcal{P}^{k}(\mathcal{O}(n))(\bar{e}) .
$$

The result follows from the fact that the sequence (2.1.3) is exact and that $\mathrm{H}^{1}(X, \mathcal{O}(n))=0$ for $n \geq 1$.

Note that by Lemma 2.2 and the sequence (2.1.3), there exists for all $1 \leq k<n$ an exact sequence of $P$-representations

$$
0 \rightarrow \mathrm{H}^{0}\left(X, \mathcal{O}(n) \otimes \mathfrak{m}_{\bar{e}}^{k+1}\right) \rightarrow \mathrm{H}^{0}(X, \mathcal{O}(n)) \rightarrow \mathcal{P}^{k}(\mathcal{O}(n))(\bar{e}) \rightarrow 0 .
$$

Since the representation $\mathrm{H}^{0}\left(X, \mathcal{O}(n) \otimes \mathfrak{m}_{\bar{e}}^{k+1}\right)$ equals $m^{k+1} S^{n-(k+1)}\left(V^{*}\right)$ as a subrepresentation of $\mathrm{H}^{0}(X, \mathcal{O}(n))=S^{n}\left(V^{*}\right)$, it follows that we have an exact sequence of $P$-representations

$$
0 \rightarrow m^{k+1} S^{n-(k+1)}\left(V^{*}\right) \rightarrow S^{n}\left(V^{*}\right) \rightarrow \mathcal{P}^{k}(\mathcal{O}(n))(\bar{e}) \rightarrow 0 .
$$


From the exact sequence

$$
0 \rightarrow m \rightarrow V^{*} \rightarrow V^{*} / m \rightarrow 0,
$$

where $m$ is the $F$-vector space on $x_{1}, \ldots, x_{N}$, we see that the representation $V^{*} / m$ is the representation corresponding to the module $L^{*}$ of $P$, giving the line bundle $\mathcal{O}(1)$ on $X=S L(V) / P$.

Lemma 2.3. For all $1 \leq k<n$ there exists a surjective map of $P$-representations

$$
\phi: S^{n}\left(V^{*}\right) \rightarrow S^{n-k}\left(L^{*}\right) \otimes S^{k}\left(V^{*}\right) .
$$

Proof. Recall that we have chosen a basis $e_{0}, \ldots, e_{N}$ for $V$, with the property that $\bar{x}_{0}$ is a basis for $L^{*}$. The $P$-representation $m$ with basis $x_{1}, \ldots, x_{N}$ gives an exact sequence

$$
0 \rightarrow m \rightarrow V^{*} \rightarrow L^{*} \rightarrow 0
$$

of $P$-representations. Define a map

$$
\phi: S^{n}\left(V^{*}\right) \rightarrow S^{n-k}\left(L^{*}\right) \otimes S^{k}\left(V^{*}\right)
$$

as follows: $\phi(f)=\bar{x}_{0}^{n-k} \otimes \partial_{0}^{n-k}(f)$, where $\partial_{0}^{n-k}$ is the $n-k$ times partial derivative with respect to the $x_{0}$-variable. Let $g$ be an element of $P$. Then by induction on the degree of the differential operator $\partial_{0}^{n-k}$ and applying the chain rule for derivation, it follows that

$$
\begin{aligned}
\phi(g f) & =\bar{x}_{0}^{n-k} \otimes \partial_{0}^{n-k}(g f)=\bar{x}_{0}^{n-k} \otimes a^{-(n-k)} g\left(\partial_{0}^{n-k} f\right) \\
& =a^{-(n-k)} \bar{x}_{0}^{n-k} \otimes g\left(\partial_{0}^{n-k} f\right)=g\left(\bar{x}_{0}^{-(n-k)} \otimes \partial_{0}^{n-k} f\right)=g \phi(f),
\end{aligned}
$$

and we see that $\phi$ is $P$-linear. It is clearly surjective, and the lemma follows.

Theorem 2.4. For all $1 \leq k<n$, the representation corresponding to $\mathcal{P}^{k}(\mathcal{O}(n))$ is $S^{n-k}\left(L^{*}\right) \otimes S^{k}\left(V^{*}\right)$.

Proof. By Lemma 2.3 there exists a surjective map of $P$-representations

$$
\phi: S^{n}\left(V^{*}\right) \rightarrow S^{n-k}\left(L^{*}\right) \otimes S^{k}\left(V^{*}\right) .
$$

We claim that $m^{k+1} S^{n-(k+1)}\left(V^{*}\right)$ equals ker $\phi$ : We first prove the inclusion

$$
m^{k+1} S^{n-(k+1)}\left(V^{*}\right) \subseteq \operatorname{ker} \phi .
$$

Pick a monomial $x_{0}^{p_{0}} x_{1}^{p_{1}} \cdots x_{N}^{p_{N}}$ in $m^{k+1} S^{n-(k+1)}\left(V^{*}\right)$; then $p_{0}+\cdots+p_{N}=n$ and $p_{0}<n-k$. These monomials form a basis for $m^{k+1} S^{n-(k+1)}\left(V^{*}\right)$. We see that $\partial_{0}^{n-k}\left(x_{0}^{p_{0}} \cdots x_{N}^{p_{N}}\right)$ is zero; hence, since $\phi$ is a linear map, it follows that we have an inclusion

$$
m^{k+1} S^{n-(k+1)}\left(V^{*}\right) \subseteq \operatorname{ker} \phi
$$

of vector spaces. The reverse inclusion follows from counting dimensions and the fact that $\phi$ is surjective: We have that

$$
\begin{gathered}
\operatorname{dim}_{F} \operatorname{ker} \phi=\operatorname{dim}_{F} S^{n}\left(V^{*}\right)-\operatorname{dim}_{F} S^{n-k}\left(V^{*}\right) \otimes S^{k}\left(V^{*}\right) \\
=\sum_{i=0}^{n}\left(\begin{array}{c}
i+N-1 \\
N-1
\end{array}\right)-\sum_{i=0}^{k}\left(\begin{array}{c}
i+N-1 \\
N-1
\end{array}\right)=\sum_{i=k+1}^{n}\left(\begin{array}{c}
i+N-1 \\
N-1
\end{array}\right),
\end{gathered}
$$


and we see that $\operatorname{dim}_{F} \operatorname{ker} \phi=\operatorname{dim}_{F} m^{k+1} S^{n-(k+1)}\left(V^{*}\right)$. It follows that

$$
m^{k+1} S^{n-(k+1)}\left(V^{*}\right)=\operatorname{ker} \phi ;
$$

hence we have an exact sequence of $P$-representations

$$
0 \rightarrow m^{k+1} S^{n-(k+1)}\left(V^{*}\right) \rightarrow S^{n}\left(V^{*}\right) \rightarrow S^{n-k}\left(L^{*}\right) \otimes S^{k}\left(V^{*}\right) \rightarrow 0 .
$$

Using sequence (2.2.1) we get isomorphisms

$$
\begin{aligned}
& \mathcal{P}^{k}(\mathcal{O}(n))(\bar{e}) \cong \mathrm{H}^{0}(X, \mathcal{O}(n)) / \mathrm{H}^{0}\left(X, \mathcal{O}(n) \otimes \mathfrak{m}_{\bar{e}}^{k+1}\right) \\
& \quad \cong S^{n}\left(V^{*}\right) / m^{k+1} S^{n-(k+1)}\left(V^{*}\right) \cong S^{n-k}\left(V^{*}\right) \otimes S^{n}\left(V^{*}\right),
\end{aligned}
$$

and it follows that $\mathcal{P}^{k}(\mathcal{O}(n))(\bar{e})$ is isomorphic to $S^{n-k}\left(L^{*}\right) \otimes S^{k}\left(V^{*}\right)$ as a representation.

Note that the result in Theorem 2.4 is true if $\operatorname{char}(F)>n$.

Corollary 2.5. For all $1 \leq k<n, \mathcal{P}^{k}(\mathcal{O}(n))$ splits as an abstract vector bundle as $\bigoplus^{\left(\begin{array}{c}N+k \\ N\end{array}\right)} \mathcal{O}(n-k)$.

Proof. Since $S^{k}\left(V^{*}\right)$ corresponds to the trivial rank $\left(\begin{array}{c}N+k \\ N\end{array}\right)$ abstract vector bundle on $\mathbf{P}\left(V^{*}\right)$, and $S^{n-k}\left(L^{*}\right)$ corresponds to the line bundle $\mathcal{O}(n-k)$, the assertion is proved.

We see that we recover results on the splitting type of the principal parts obtained in [6], [7, 8] and [9].

\section{ACKnowledgments}

I would like to thank Michel Brion for an invitation to spend spring 2001 at the Institut Fourier. Thanks for suggesting the problem, sharing ideas and for answering numerous questions on homogeneous spaces and representation theory. Thanks also to Laurent Manivel for stimulating discussions on the subject. I would also like to thank Mina Teicher for an invitation to the Bar-Ilan University, where parts of this work were done and this note was written. Finally, thanks to Dan Laksov for comments.

\section{REFERENCES}

[1] D. N. Akhiezer, Lie group actions in complex analysis, Aspects of Mathematics, Vieweg, Braunschweig, 1995. MR 96g:32051

[2] A. Borel, Linear algebraic groups, Graduate Texts in Mathematics, no. 126, Springer-Verlag, New York, 1991. MR 92d:20001

[3] F. Cukierman, Determinant of complexes and higher Hessians, Math. Ann. 307, 225-251 (1997). MR 98d:14067

[4] J. C. Jantzen, Representations of algebraic groups, Pure and Applied Mathematics, no. 131, Academic Press, 1987. MR 89c:20001

[5] R. Hartshorne, Algebraic geometry, Graduate Texts in Mathematics, no. 52, Springer-Verlag, New York and Heidelberg, 1977. MR 57:3116

[6] H. Maakestad, Modules of principal parts on the projective line, preprint math.AG/0111149.

[7] D. Perkinson, Principal parts of line bundles on toric varieties, Compositio Math. 104, 27-39 (1996). MR 98b:14039

[8] R. Piene and G. Sacchiero, Duality for rational normal scrolls, Comm. Algebra 12, 1041-1066 (1984). MR 86c:14042 
[9] S. di Rocco and A. J. Sommese, Line bundles for which a projectivized jet bundle is a product, Proc. Amer. Math. Soc. 129, no. 6, 1659-1663 (2001). MR 2002c:14012

[10] A. J. Sommese, Compact complex manifolds possessing a line bundle with a trivial jet bundle, Abh. Math. Sem. Univ. Hamburg 47, 79-91 (1978). MR 58:17231

Department of Mathematics, Bar-Ilan University, Ramat Gan, Israel

E-mail address: makesth@macs.biu.ac.il

Current address: Department of Mathematics, KTH, 10044 Stockholm, Sweden 